\title{
Does Coinoculation with Different Verticillium dahliae Genotypes Affect the Host or Fungus?
}

\author{
David Linnard Wheeler ${ }^{\dagger}$ and Dennis Allen Johnson
}

Department of Plant Pathology, Washington State University, Pullman, WA, U.S.A.

Accepted for publication 2 January 2019.

\begin{abstract}
Inferences about Verticillium dahliae are often deduced from experiments where hosts are inoculated with one isolate. The assumption that the outcomes from these experiments scale with $V$. dahliae diversity is untested. The objectives of this research were to test the hypotheses that (i) coinoculation with combinations of isolates affects plant biomass, disease expression, and fungal colonization; and (ii) hosts select for the specific isolates. Potato, mustard, and barley plants were coinoculated with seven combinations of three isolates. Genotypes recovered from infected plants were genotyped with microsatellite markers. Disease expression and fungal colonization but not plant biomass of potato was affected by coinoculation

$(F=7.07, P<0.0001 ; F=2.36, P=0.0427)$ and depended on the isolates with which plants were inoculated. One genotype was disproportionately selected for by all hosts. Putative heterokaryons were recovered from mustard plants coinoculated with isolates of different vegetative compatibility groups (VCG). These results support the assumption that mixed infections have marginal impacts on plant biomass but challenge the assumption that they do not affect disease expression and fungal colonization. Finally, this research provides evidence that plants select for specific $V$. dahliae genotypes and isolates from different VCGs can anastomose in planta.
\end{abstract}

Verticillium wilts are vascular diseases caused by fungi in the genus Verticillium. Within this genus, Verticillium dahliae Kleb. causes wilts of several hundred plants species (Berlanger and Powelson 2000) and no symptoms on many other hosts (Malcolm et al. 2013; Wheeler and Johnson, 2016). In the Pacific Northwest of the United States, Verticillium wilt of potato (Solanum tuberosum L.) is especially problematic and can cause yield losses up to $50 \%$ (Powelson and Rowe 1993). Additionally, potato crops are often grown in fields where other symptomatic hosts, including mint (Mentha spp. L.), and asymptomatic hosts, including mustards (e.g., Brassica spp. L.) and grasses (e.g., Hordeum vulgare L.), are cultivated. The wide host range of $V$. dahliae together with the production of long-lived microsclerotia enable this fungus to persist in infested fields for extended periods of time between crop rotations (Wilhelm 1955).

The cultivation of symptomatic and asymptomatic hosts of $V$. dahliae within close proximity or even within the same fields has likely contributed to the maintenance of relatively diverse populations within a shared geographical area (Dung et al. 2013; Wheeler et al. 2019). Although $V$. dahliae reproduces mitotically and most populations are clonal (Dung et al. 2013; Short et al. 2015), this fungus possesses conserved genes involved in sexual reproduction in other fungi, including both mating type idiomorphs (Short et al. 2014; Usami et al. 2009). Furthermore, sexual recombination has been inferred (Milgroom et al. 2014) but not observed directly. Despite the primarily asexual reproductive mode of $V$. dahliae, genotypic and phenotypic diversity exist (Klosterman et al. 2009, 2011).

${ }^{\dagger}$ Corresponding author: D. L. Wheeler; E-mail: david.wheeler@wsu.edu

Funding: We thank the Northwest Potato Research Consortium for providing financial support of this research, PPNS number 0764, Department of Plant Pathology, College of Agricultural, Human, and Natural Resource Sciences, Agricultural Research Center, Hatch Project number WNP00678, Washington State University, Pullman, WA 99164-6430, U.S.A.

The author(s) declare no conflict of interest.

C 2019 The American Phytopathological Society
Historically, phenotypic and genotypic diversity have been characterized with nonself-recognition assays and molecular markers. Specifically, diversity is generally characterized with vegetative compatibility assays, where auxotrophic mutants of isolates are assigned to one of five vegetative compatibility groups (VCGs) after anastomosis and heterokaryosis with tester strains (Joaquim and Rowe 1990; Milgroom 2015). VCGs are useful because they are often associated with pathogenicity (Katan 2000); however, they are not always concordant with clonal lineages (Jiménez-Gasco et al. 2014). Hence, molecular markers have been employed to document allelic and genotypic diversity of $V$. dahliae among countries (Korolev et al. 2001) and regions (Rafiei et al. 2018) and within fields (Gurung et al. 2014; Jiménez-Díaz et al. 2011).

Despite the cooccurrence of $V$. dahliae diversity within fields (Gurung et al. 2014; Jiménez-Díaz et al. 2011), most studies have used single isolates to make inferences about this fungus (Wheeler and Johnson 2016). Of the published reports where plants were coinoculated with more than one Verticillium sp. or isolate, results vary and depend on ambient temperature (Matta and Garibaldi 1977), the method of inoculation, the order in which each species is used to inoculate the host, and the time between inoculations (Price and Sackston 1989). Overall, the impacts of coinoculation with different species of Verticillium vary from no observed effects (Matta and Garibaldi 1977) to cross-protection, where symptoms are reduced by sequential or simultaneous inoculation of nonaggressive isolates or species (Davis and Sorensen 1985; Matta and Garibaldi 1977; Qin et al. 2008; Robinson et al. 2007; Tyvaert et al. 2014). Within the species $V$. dahliae, less variation was observed and only cross-protection was reported (Barrow 1969; Price and Sackston 1983; Shittu et al. 2009). These results provide valuable insight about the impacts of fungal inter- and intrageneric interactions on host plants; however, most claims about crossprotection are derived from artificial experimental settings that do not reflect biological reality. Specifically, the isolates selected in some cases were weakly aggressive and inoculated sequentially with conidia, not microsclerotia.

The primary objective of this research was to test the hypothesis that coinoculation of potato, mustard, and barley with different combinations of three $V$. dahliae isolates affects tuber yields, 
mustard and barley biomass, symptom expression of potato, and fungal colonization. A corollary objective was to test the hypothesis that these hosts systematically select for specific $V$. dahliae genotypes. Potato, mustard, and barley plants were grown in soilless potting media infested with different combinations of microsclerotia from three $V$. dahliae isolates. Potato yields, mustard and barley biomass, wilt symptoms, and fungal colonization were quantified and compared. To determine whether these hosts select for specific genotypes of $V$. dahliae, genotypes recovered from hosts were genotyped with polymorphic microsatellite markers.

\section{MATERIALS AND METHODS}

Effects of coinoculation on potato yields, mustard and barley biomass, disease expression, and fungal colonization. The impacts of coinoculation on plant yields, disease expression, and fungal colonization were determined with a greenhouse experiment. This experiment comprised a two-way factorial treatment structure with three plant species, seven combinations of three $V$. dahliae isolates, five replicates, and two trials. The isolates for each of the treatments are described in Table 1. To determine the effects of coinoculation on both symptomatic and asymptomatic hosts, one symptomatic host species (potato Russet Burbank [S. tuberosum L.]) and two asymptomatic host species (brown mustard ISCI 99 [Brassica juncea L.] and barley Baroness $[H$. vulgare L.]) were selected. To determine the responses of these plant species to coinoculation with isolates that vary in aggressiveness, three such isolates were selected (Dung et al. 2013). Potted plants were treated as biological replicates and experimental units. Replicates were arranged perpendicular to a temperature gradient in a randomized complete block design structure.

Before plants were grown, soilless potting media (Sun Gro Horticulture, Agawam, MA, U.S.A.) was infested with $V$. dahliae at $20 \mathrm{CFU} / \mathrm{cm}^{3}$, as described by Wheeler and Johnson (2016). For each treatment, the amount of inoculum used to infest each experimental unit was determined by dividing $20 \mathrm{CFU} / \mathrm{cm}^{3}$ by the number of isolates used per treatment. For example, treatments comprising two isolates were infested with each isolate at $10 \mathrm{CFU} /$ $\mathrm{cm}^{3}$ for a total inoculum density of $20 \mathrm{CFU} / \mathrm{cm}^{3}$ of soilless potting media.

Potato, mustard, and barley were subsequently planted in infested or noninfested soilless potting media in 3-liter pots (J. M. McConkey \& Co., Inc., Sumner, WA, U.S.A.). Plants were grown in greenhouses with photoperiods between 12 and $15 \mathrm{~h} /$ day, daytime temperatures between 13 and $29^{\circ} \mathrm{C}$, and nighttime temperatures between 11 and $27^{\circ} \mathrm{C}$.

Upon emergence of potato plants, symptoms were monitored every week after 3 weeks until plant senescence. For potato plants, disease severity was estimated and expressed as the percentage of the whole plant that exhibited chlorosis or necrosis. Disease severity data were used to calculate the area under the senescence progress curve, as described by Shaner and Finney (1977). Upon senescence, tuber yield or plant biomass was quantified for each species. For potato, yields were estimated and expressed as the weight of all tubers that weighed more than $20 \mathrm{~g} / \mathrm{tuber}$. For mustard and barley plants, dried aboveground biomass was weighed and recorded.

Fungal colonization was quantified for each plant after senescence. Dried plant stems were ground and weighed, and five technical replicates of $0.1 \mathrm{~g}$ were plated on the surface of semiselective NP-10 media (Kabir et al. 2004), as described by Wheeler and Johnson (2016). Plates were incubated for 14 days in the dark at $22^{\circ} \mathrm{C}$ before stem debris was rinsed from the surface of each plate and Verticillium-like colonies were counted with a dissecting scope. Mean stem inoculum density (CFU per gram) was calculated by averaging values from five technical replicates within each biological replicate.
Differences in yields or biomass, disease expression, and fungal colonization between treatments were detected with analysis of variance. Separate models were fit for each crop. Assumptions of normality, homoscedasticity, and independence were evaluated with boxplots, quantile-quantile plots, and residual versus fitted values plots. Isolates were treated as fixed effects while blocks and trials were treated as random effects. Posthoc comparisons were calculated with Fisher's least significant difference test with the 'agricolae' package (de Mendiburu 2017) in R.

Selection of $\boldsymbol{V}$. dahliae genotypes by hosts. To differentiate the genotypes recovered from each plant species, at maximum, three $V$. dahliae colonies were subsampled from each biological replicate of every species and genotyped with two polymorphic microsatellites (Almany et al. 2009). Colonies recovered from inoculated plants are henceforth referred to as "genotypes" to enable differentiation from the "isolates" used to inoculate each crop.

Upon senescence of each plant species and after $V$. dahliae was quantified from dried stems, three colonies of $V$. dahliae were selected from separate technical replicates within each biological replicate. From each of the three colonies recovered from separate technical replicates, a single mass of conidia was harvested from an individual phialide of the verticillate conidiophores with the sharp and narrow splinter of a broken and sterilized toothpick. Thus, in total, at maximum, three colonies were recovered from each biological replicate. These masses of conidia were then transferred to NP-10 media and subcultured until no contaminates were detected. Each subsampled colony was transferred to potato dextrose agar overlaid with two layers of dialysis membrane (Spectrum Laboratories, Rancho Dominguez, CA, U.S.A.) and incubated in the dark for 15 days at $22^{\circ} \mathrm{C}$. Mycelia were harvested and lyophilized, and DNA was extracted with the glass bead breakage method described by Dobinson (1995). Genomic DNA quantities were determined by comparing the width and intensity of sample bands with known $\lambda$ DNA standards on agarose gels.

Subsampled colonies from each biological replicate were genotyped with two polymorphic microsatellite markers (Almany et al. 2009) to differentiate isolates. Specifically, each subsample was genotyped with primers for VD8 and VD10 loci. Polymerase chain reaction (PCR) for these loci was performed as described by Wheeler et al. (2019). Differences in PCR amplicon size were determined thrice on 3\% (wt/vol) agarose gels. At the VD8 locus, isolate 461 was differentiated from 653 and 111 by $\geq 66$ bp differences but 653 and 111 were indistinguishable. At the VD10 locus, isolate 111 was differentiated from 653 and 461 by $\geq 92$ bp differences but 653 and 461 were indistinguishable. Together, the pairwise differences in amplicon sizes between isolates at these loci enabled differentiation of these isolates.

For putative heterokaryons that produced two amplicons at both loci on agarose gels, fragment sizes were corroborated with capillary electrophoresis on an ABI 3730xl DNA Analyzer (Applied Biosystems, Foster City, CA) at the United States Department of Agriculture Wheat Genetics, Quality, Physiology, and Disease Research Lab. After PCR, $3 \mu$ of PCR products were first diluted with $22 \mu \mathrm{l}$ of sterilized and distilled water. A total of $3 \mu \mathrm{l}$

TABLE 1. Coinoculation treatments comprising different combinations of three Verticillium dahliae isolates collected from potato, peppermint, and tomato $^{\mathrm{a}}$

\begin{tabular}{lccccc}
\hline Isolate & Host & VCG & Mating type & Haplotype & \multicolumn{1}{c}{ Source } \\
\hline 653 & Potato & 4A & MAT1-2 & H04 & R. Rowe \\
111 & Mint & 2B & MAT1-2 & H02 & D. Johnson \\
461 & Tomato & 2 & $M A T 1-1$ & H37 & R. Rowe \\
653 and 111 & $\ldots$ & $\ldots$ & $\ldots$ & $\ldots$ & $\ldots$ \\
653 and 461 & $\ldots$ & $\ldots$ & $\ldots$ & $\ldots$ & $\ldots$ \\
111 and 461 & $\ldots$ & $\ldots$ & $\ldots$ & $\ldots$ & $\ldots$ \\
653,111, and 461 & $\ldots$ & $\ldots$ & $\ldots$ & $\ldots$ & $\ldots$ \\
\hline
\end{tabular}

a Isolates varied in vegetative compatibility group (VCG), mating type idiomorphs, and multilocus haplotypes (Dung et al. 2013). 
of diluted PCR product was then mixed with $3 \mu$ of LIZ-685 size standard and $7 \mu \mathrm{l}$ of Hi-Di formamide (Applied Biosystems). The resultant solution was denatured at $95^{\circ} \mathrm{C}$ for $5 \mathrm{~min}$. After capillary electrophoresis, fragments sizes were scored in GeneMarker (SoftGenetics LLC, State College, PA, U.S.A.).

To further corroborate or refute the presence of heterokaryons, the mating type idiomorphs were amplified and compared across samples. PCR was completed as described by Usami et al. (2009). Mating types were compared on $1 \%$ (wt/vol) agarose gels where isolates with MAT1-1 produced approximately 400-bp fragments and MAT1-2 produced approximately 600-bp fragments (Usami et al. 2009). Each putative heterokaryon or aneuploid was assayed thrice.

\section{RESULTS}

Effects of coinoculation on potato yields, mustard and barley biomass, disease expression, and fungal colonization. Potato yields and mustard and barley biomass were not affected by coinoculation of the three $V$. dahliae isolates (Fig. 1). Specifically, potato yields were not different among coinoculated treatments but yields of the noninfested control were greater $(F=4.84, P=$ 0.0002) than all of the inoculated controls. Similarly, biomass of mustard $(F=1.75, P=0.1143)$ and barley $(F=0.23, P=0.9753)$ were not affected by coinoculation (Fig. 1).

Disease expression of potato plants was affected $(F=7.07, P<$ 0.0001 ) by both single and coinoculations and was sensitive to the isolates used for inoculations (Fig. 2). For the plants inoculated with single isolates, disease severity was greatest in plants inoculated with isolate 653, moderate in plants inoculated with 111 , and least in plants inoculated with 461 . For the plants coinoculated with two or three isolates, disease expression was most severe in plants inoculated with 653, 653 and 111, and all three isolates. Disease was least severe in plants inoculated with 461 . Disease was slightly more severe in plants inoculated with all three isolates or in plants inoculated with 653 and 111 relative to the other coinoculated treatments. Disease severity increased in potato plants inoculated with 111 or 461 when coinoculated with 653 relative to those plants only inoculated with 111 or 461 (Fig. 2).

Fungal colonization of potato $(F=2.36, P=0.0427)$ but not mustard $(F=1.56, P=0.1771)$ and barley were affected by coinoculation (Fig. 3). Specifically, for the plants inoculated with single isolates, colonization was greater in plants inoculated with 461 than with 653 or 111 . For the plants coinoculated with two or three isolates, colonization was marginally greater in plants coinoculated with 461 and 653 relative to other treatments. Overall, isolate 461 caused low levels of extensively colonized potato stems relative to other treatments, except when coinoculated with 111 or 653 (Fig. 3).

Selection of $\boldsymbol{V}$. dahliae genotypes by hosts. The recovery of genotypes from each host varied across treatments (Fig. 4). All genotypes of V. dahliae $(653,111$, and 461) were recovered from all coinoculated potato and mustard treatments but not from barley plants. Only genotype 461 was recovered from inoculated barley plants. A subset of genotypes was recovered from most plants. In mustard plants, however, all genotypes were recovered from different individual plants inoculated with all three isolates (Fig. 4).

Of the coinoculated potato, mustard, and barley plants, all three genotypes were not recovered from any individual plants (Fig. 5). Two genotypes, however, were recovered from approximately $30 \%$ of individual coinoculated plants and one genotype was recovered from approximately $30 \%$ of individual coinoculated plants. For potato plants coinoculated with two or three isolates, two genotypes were recovered from the stems of $40 \%$ of individual plants. Similarly, for mustard plants coinoculated with two or three isolates, two genotypes were recovered from the stems of $60 \%$ of individual plants (Fig. 5).

Differences in the frequencies of recovered genotypes were observed from plants coinoculated with multiple $V$. dahliae isolates (Fig. 4). Specifically, for potato and mustard plants coinoculated with any combination of isolates that included 461, this genotype was recovered at numerically higher frequencies than the other

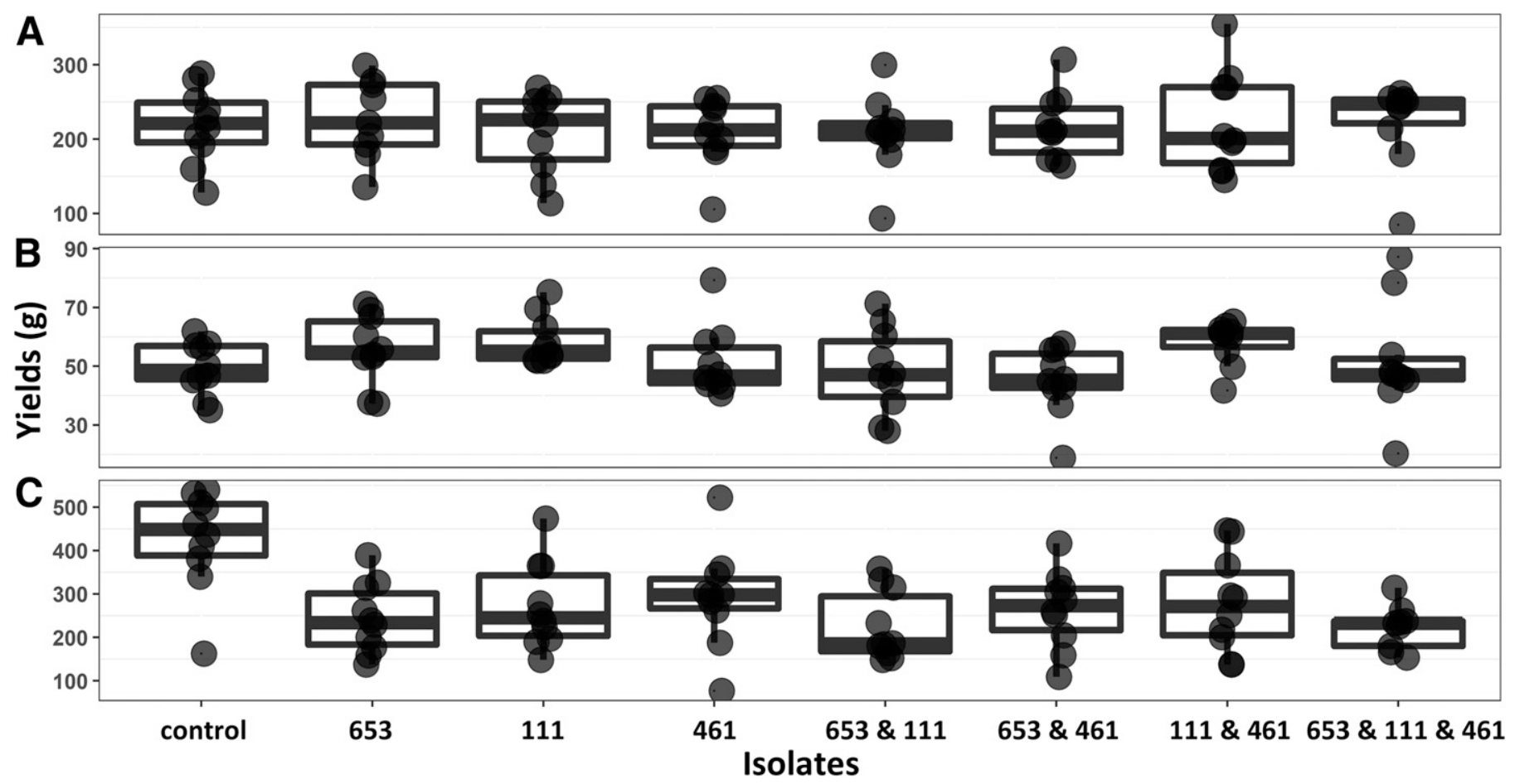

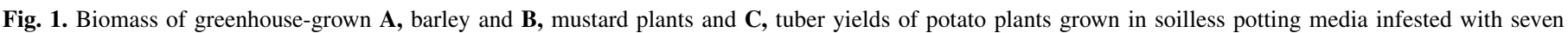

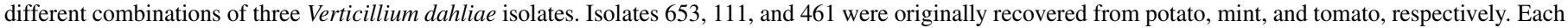
dot represents an observation and boxplots summarize the distribution of each treatment. 
genotypes (Fig. 4). Conversely, for potato and mustard plants coinoculated with 653 and 111, approximately half of each genotype was recovered from infected stems.

In addition to the one or two individual genotypes that were recovered from each host, putative heterokaryons that produced two amplicons at one microsatellite locus were also recovered from five different individual mustards plants (Fig. 4). The presence of two alleles at VD8 and VD10 was corroborated with capillary electrophoresis, where two peaks were observed for each isolate. In total, five putative heterokaryons were recovered from

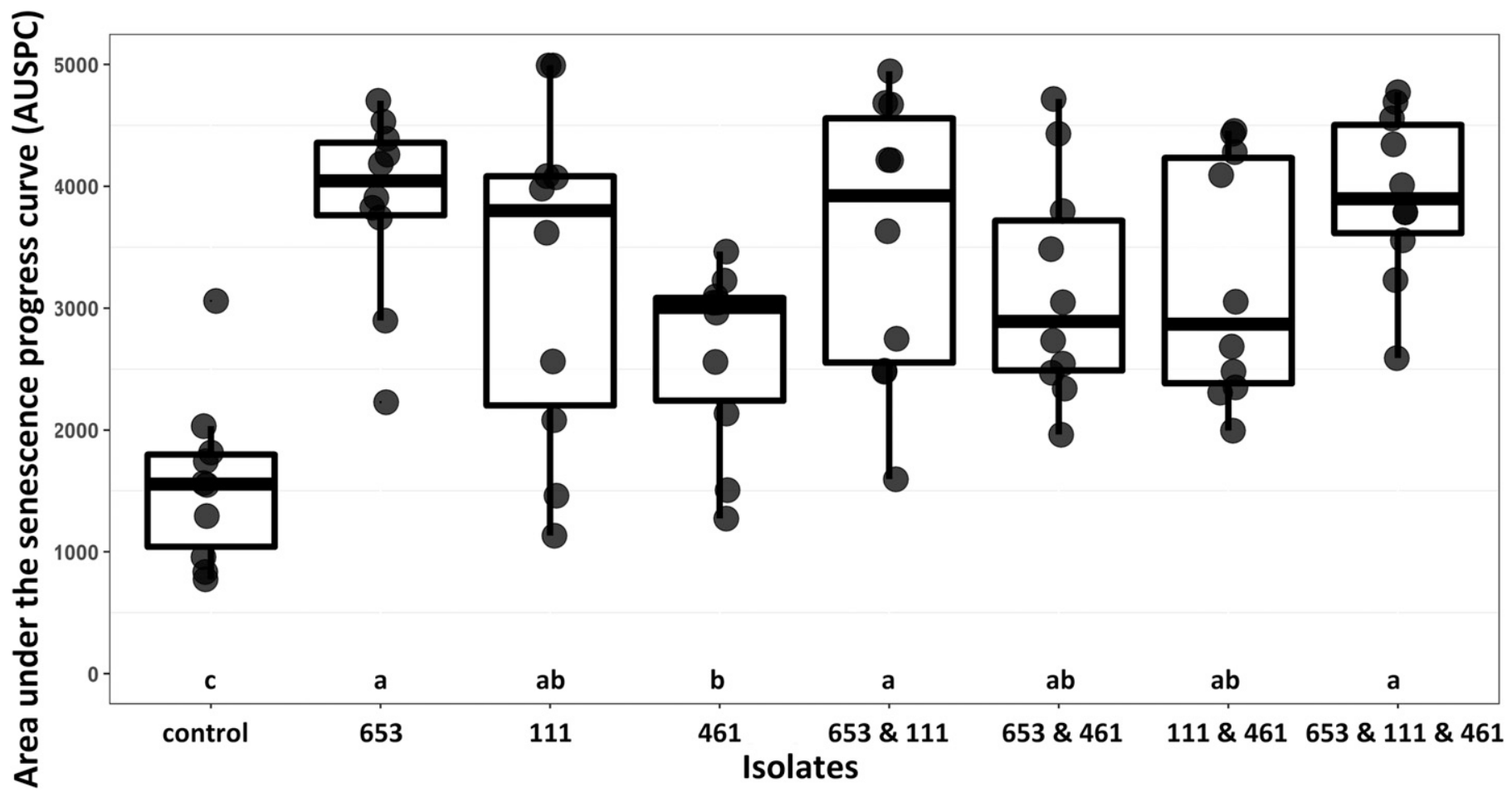

Fig. 2. Disease symptoms of greenhouse-grown potato plants, expressed as the area under the senescence progress curve, grown in soilless potting media infested with seven different combinations of three Verticillium dahliae isolates. Isolates 653, 111, and 461 were originally recovered from potato, mint, and tomato, respectively. Dots represent observations and boxplots summarize the distributions of the treatments. Letters along the top of the x-axis were assigned with Fisher's least significant difference $=815.12$ posthoc test. Treatments with different letters are significantly different $(P<0.05)$.

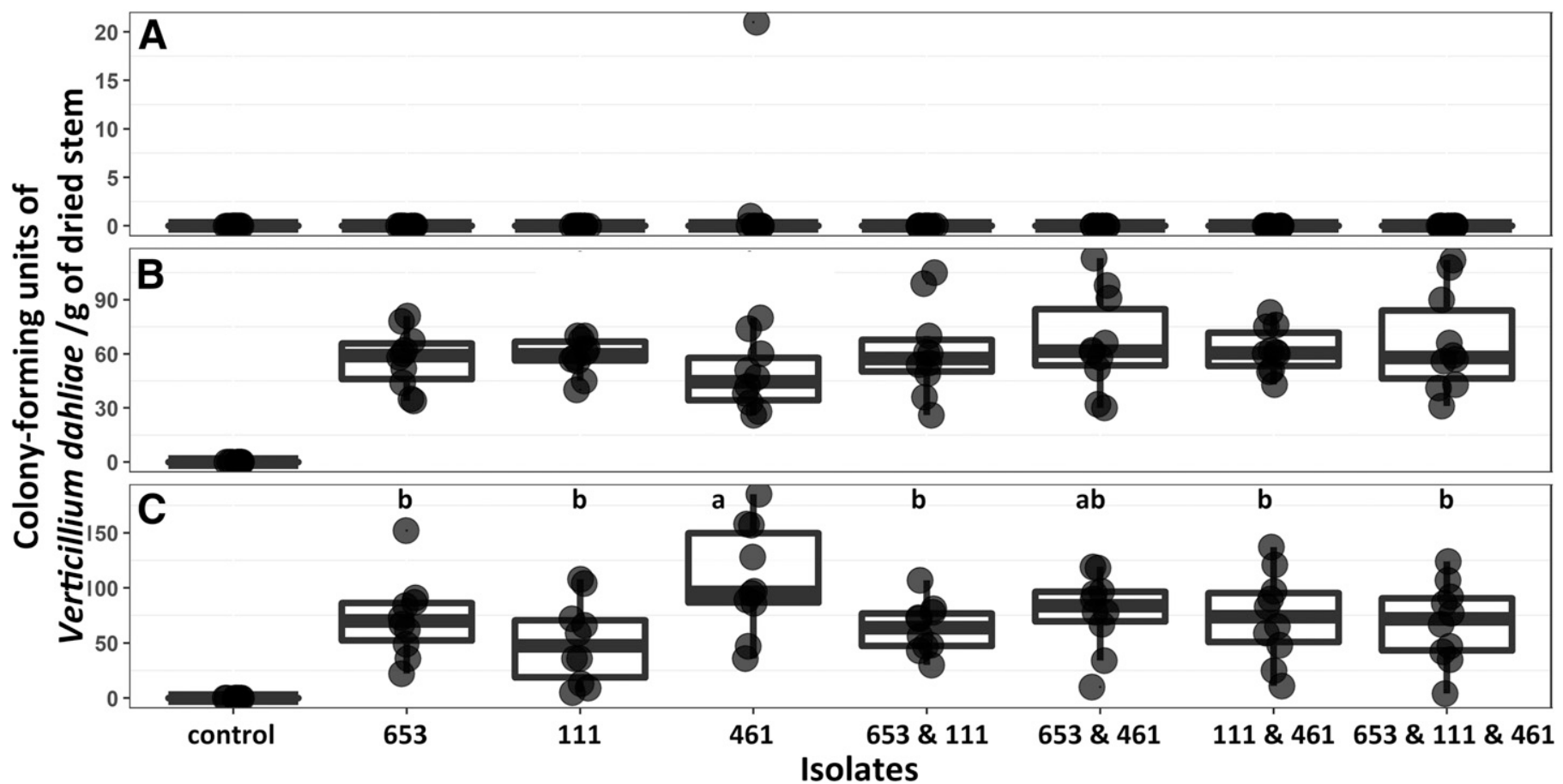

Fig. 3. CFU of Verticillium dahliae/g of dried stems recovered from greenhouse-grown A, barley; B, mustard; and $\mathbf{C}$, potato plants grown in soilless potting media infested with seven different combinations of three $V$. dahliae isolates. Isolates 653,111 , and 461 were originally recovered from potato, mint, and tomato, respectively. Dots represent observations and boxplots summarize the distributions of the treatments. Letters above the boxplots for potato were assigned with Fisher's least significant difference $=32.38$ posthoc test. Treatments with different letters are significantly different $(P<0.05)$. 
coinoculated mustard plants. Specifically, heterokaryons with alleles from genotypes 653 and 461 were recovered from plants inoculated with all three isolates $(n=2)$ and plants inoculated with 653 and $461(n=1)$. Similarly, heterokaryons with alleles from genotypes 111 and 461 were recovered from two plants. In addition to possessing two alleles at VD8 and VD10, these five putative heterokaryons also possessed both mating type idiomorphs, MAT1-2 from 653 or 111 and MAT1-1 from 461.

\section{DISCUSSION}

The fungus $V$. dahliae is a pathogen and endophyte of many plant species that exists in relatively diverse populations within fields (Gurung et al. 2014; Jiménez-Díaz et al. 2011). Despite the awareness that different isolates of $V$. dahliae coexist within fields, many studies extrapolate results from simplified experiments to field settings. The authors are among this group. The assumption that individual host-plant interactions scale with increased complexity may or may not be justifiable and likely depends on a variety of factors, including the organisms involved. The results reported herein lend credence to and challenge this assumption. Mixed infections of up to three $V$. dahliae isolates, for example, did not affect plant yields or biomass, at least for potato, mustard, and barley. Conversely, coinoculations affected disease expression and fungal colonization of potato. Finally, the differential recovery of different $V$. dahliae genotypes from coinoculated plants and the formation of putative heterokaryons from vegetatively incompatible isolates challenge this assumption.

Tuber yields of potato and biomass of mustards and grasses were unresponsive to coinoculations. Although this is a "negative result",
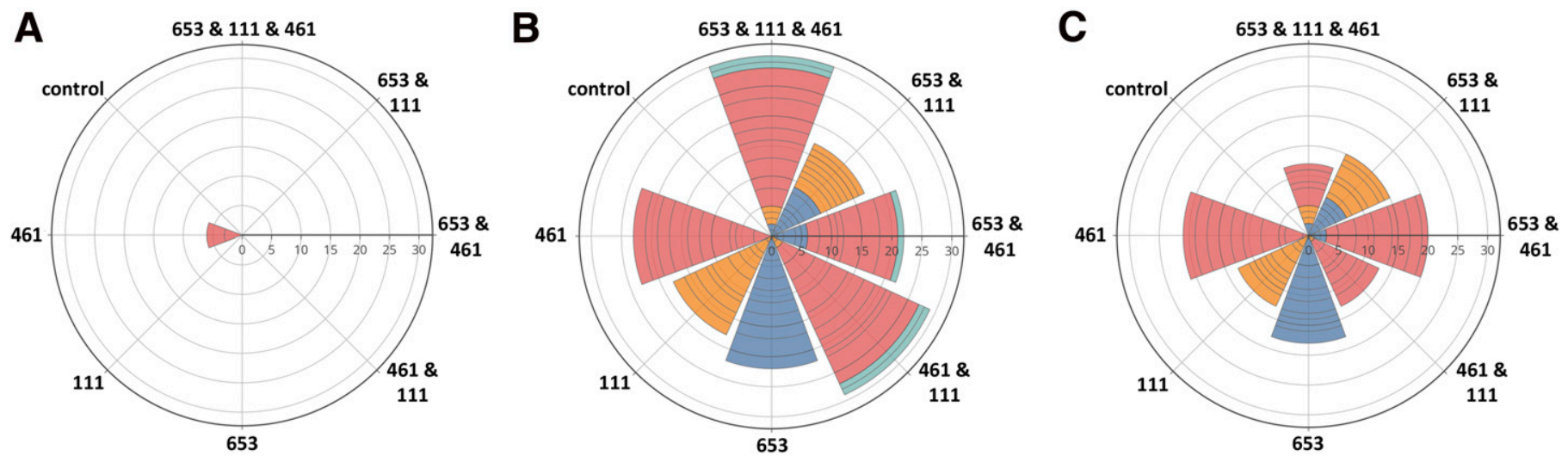

Fig. 4. Frequencies, expressed as proportions of three Verticillium dahliae isolates recovered from greenhouse-grown A, barley; $\mathbf{B}$, mustard; and $\mathbf{C}$, potato plants grown in soilless potting media infested with seven different combinations of three $V$. dahliae isolates. Isolates 653,111 , and 461 were originally recovered from potato, mint, and tomato, respectively. Genotypes of subsampled $V$. dahliae colonies recovered from plants were determined by identifying the size of alleles at two microsatellite loci with gel and capillary electrophoresis. Triangular bars represent the total counts of each isolate. Counts were calculated by summing the number of each isolate recovered from three subsampled colonies collected from each biological replicate. The radial axes represent counts of 653 (blue), 111 (orange), 461 (red), and putative heterokaryons (teal) recovered from infected stems.

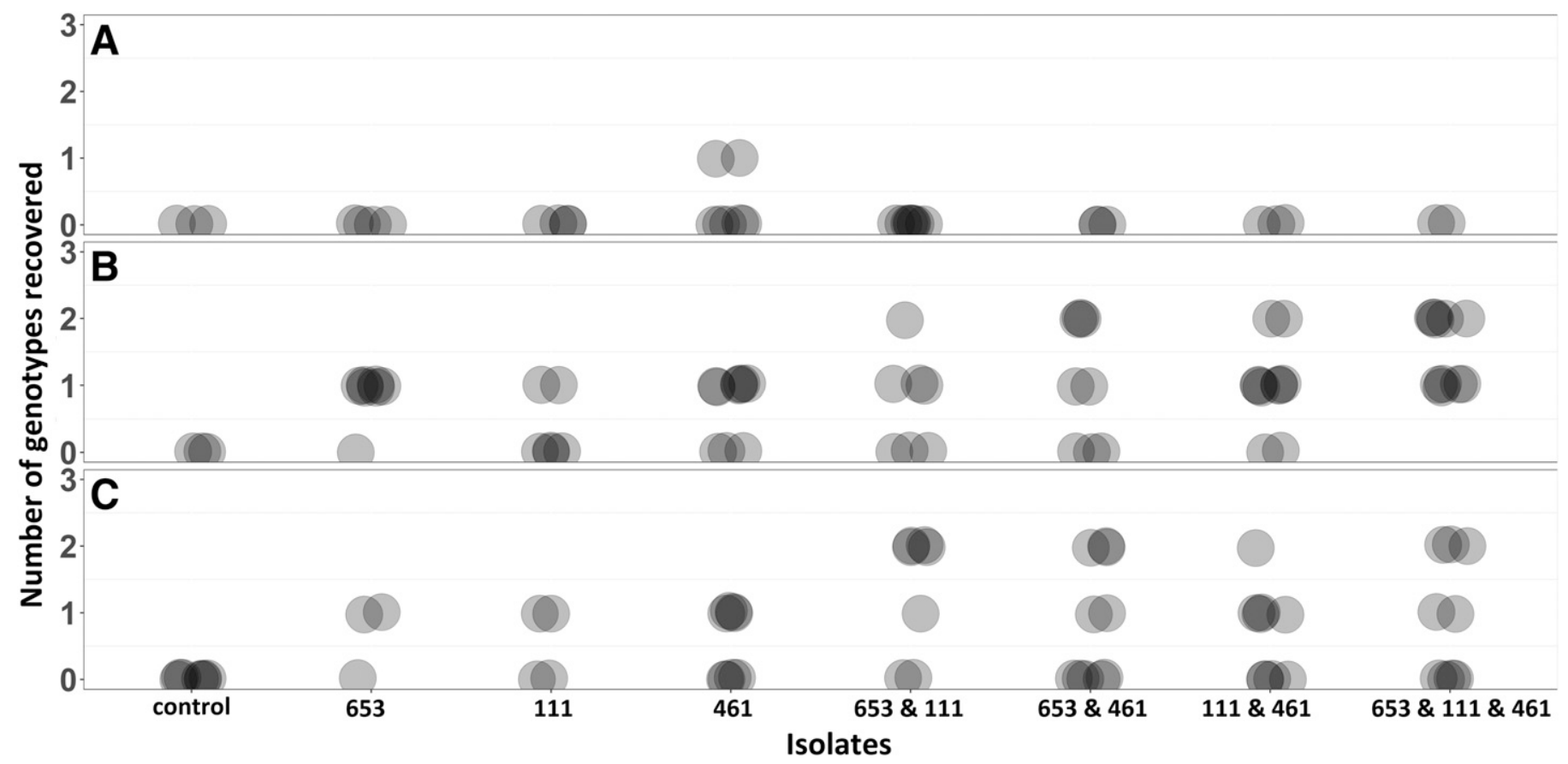

Fig. 5. Numbers of Verticillium dahliae genotypes that were recovered from individual greenhouse-grown A, barley; $\mathbf{B}$, mustard; and $\mathbf{C}$, potato plants grown in soilless potting media infested with seven different combinations of three V. dahliae isolates. Isolates 653 , 111, and 461 were originally recovered from potato, mint, and tomato, respectively. Dots represent observations calculated by summing the total number of different isolates recovered from subsamples for each biological replicate. 
it stands in contrast to most (Davis and Sorensen 1985; Price and Sackston 1983; Shittu et al. 2009) but not all (Qin et al. 2008) reports where investigators observed differences in plant height or yields in response to coinoculations. The sources of this discrepancy are likely (i) the species or isolates used, (ii) the timing, and (iii) the methods of inoculation among studies. In most studies, weakly aggressive isolates or different species were used to inoculate a host prior to pathogen inoculation using artificial techniques such as stem injection, root dips, or soil drenches (Davis and Sorensen 1985; Matta and Garibaldi 1977; Price and Sackston 1983; Shittu et al. 2009; Tyvaert et al. 2014; Qin et al. 2008). Regardless of the sources of the discrepancy between the results reported here and existing reports, these results corroborate the expectation that mixed infections of pathogenic $V$. dahliae isolates do not affect plant biomass.

Although coinoculations did not affect plant biomass, disease expression of potato plants was affected by coinoculations. Specifically, symptoms were reduced in plants inoculated with 461 and one other isolate unless two other isolates, 653 and 111, were coinoculated. This observation, although derived from just three isolates of $V$. dahliae, exposes the nuanced effects of multiple isolates on disease expression. Although differences in disease expression induced by coinoculation of $V$. dahliae isolates have been reported before (Price and Sackston 1983; Shittu et al. 2009), these studies use artificial inoculation methods to induce crossprotection with weakly aggressive isolates. In contrast, our results reflect, albeit in a simplified system, the commonplace interactions that might occur between plants grown in soil infested with a couple of different pathogenic isolates of $V$. dahliae. Moreover, these results underscore the importance of the potential impacts of intraspecific diversity on disease expression, especially in field settings where different genotypes of $V$. dahliae cooccur (Gurung et al. 2014; Jiménez-Díaz et al. 2011).

Like disease expression, fungal colonization of potato (but not mustard or barley) was also affected by coinoculation. In contrast to disease expression, isolate 461 produced more CFU on potato than other treatments, except for plants coinoculated with 653 and 461. Surprisingly, the amount of CFU produced on potato stems by 461 alone was mitigated when 461 was coinoculated with 111 or with both 653 and 111 . These results corroborate differences in fungal colonization observed by Shittu et al. (2009) and establish that these differences can be induced by different pathogenic genotypes within $V$. dahliae and not exclusively by weakly aggressive isolates. This observation is also important for (i) fellow researchers who might want to consider the influence of multiple genotypes on stem infection when designing field experiments in infested field soils, and (ii) growers who might interpret counts of $V$. dahliae from soil with caution depending on the genotypes present in a given field.

Along with the differences in fungal colonization that were observed across coinoculated plants, differences in the number of genotypes recovered from each species and the proportion of each genotype recovered from each host were observed. Although all genotypes were recovered from different individual potato and mustard plants, only one or two unique genotypes were recovered from the same individual plants. More specifically, genotype 461 was recovered at higher frequencies than 653 or 111 in coinoculated plants and in barley, where it was the only genotype recovered. These differences in the frequency of each genotype might be explained by increased fitness of 461 relative to other isolates or differential susceptibility of the hosts. For example, $461 \mathrm{might}$ infect plants before other isolates, induce plant defense responses, and, thereby, terminate all subsequent infection courts. This explanation is consistent with hypotheses posited by Robinson et al. (2007), Qin et al. (2008), Shittu et al. (2009), and Tyvaert et al. (2014) but is inconsistent with our observation that up to two unique genotypes, including 461, were recovered from some individual plants. Alternatively, plants were likely infected by several isolates but 461 reached stem tissues or grew out of sampled stems faster than other genotypes. Coinoculations of isolates with fluorescently labeled nuclei sampled over time could be used to determine the ways in which certain isolates outcompete others. Regardless of the mechanisms involved in the differential recruitment or fungal colonization of one isolate over another, this observation has potential explanatory importance. For example, in a scenario where field soil is infested with at least two $V$. dahliae genotypes that differ in aggressiveness, it follows that specific asymptomatic hosts such as mustard might be planted to select for the least aggressive genotype and, thereby, over several years, change the composition of genotypes and reduce disease expression in susceptible crops such as potato.

Putative heterokaryons were recovered from five different mustard plants coinoculated with vegetatively incompatible isolates. These are putative heterokaryons because, although they possess two alleles at two microsatellite loci and both mating-type idiomorphs, the authors do not know if different nuclei share a common cytoplasm within a cell or if karyogamy occurred and the resultant nucleus is an aneuploid or diploid. To determine whether these genotypes are stable heterokaryons or transient and form aneuploid or diploid nuclei, different isolates could be labeled with fluorescent proteins, coinoculated, and inspected in mustard plants across time. This approach has been used in vitro with the related fungus Colletotrichum lindemuthianum (Ishikawa et al. 2012). Although it is perhaps counterintuitive that isolates from different VCGs can anastomose, this observation is not unprecedented. For example, parasexual recombination has been documented within (Hastie 1964) and between Verticillium spp. (Hastie 1973) under laboratory conditions and stable diploid formation has been inferred between species under field conditions (Inderbitzin et al. 2011). In addition, evidence of sexual recombination between isolates from different VCGs has been detected with phylogenetic approaches (Jiménez-Gasco et al. 2014; Milgroom et al. 2014). Regardless of the nuclear condition of these genotypes, the role of heterokaryosis in introducing genetic diversity during the lifetime of a fungus (Strom and Bushley 2016) and the impact on host plants deserves further investigation.

This research provides a valuable contribution to our collective understanding of $V$. dahliae ecology, the effects of mixed infections on both the host and the fungus, and intraspecific interactions among isolates. Although this research represents a step toward understanding the relationships among $V$. dahliae genotypes and their impacts on plants, the authors do not pretend that this research is exhaustive and representative of the diversity of interactions that occur in field settings. Finally, we hope that this research motivates future research on the intraspecific interactions, heterokaryon formation, and impacts of these phenomena on plant disease management.

\section{LITERATURE CITED}

Almany, G. R., De Arruda, M. P., Arthofer, W., Atallah, Z. K., Beissinger, S. R., Beruman, M. L., Bogdanowicz, S. M., Brown, S. D., Bruford, M. W., Burdine, C. 2009. Permanent genetic resources added to molecular ecology resources database. Mol. Ecol. Resour. 9:1460-1466.

Barrow, J. R. 1969. Cross-protection against field infestation of Verticillium wilt. Pages 32-33 in: Proc. Beltwide Cotton Prod. Res. Conf. New Orleans, LA, U.S.A..

Berlanger, I., and Powelson, M. L. 2000. Verticillium wilt. Plant Health Instruct. doi.org/10.1094/PHI-I-2000-0801-01

Davis, J. R., and Sorensen, L. H. 1985. Association of Verticillium tricorpus with Verticillium dahliae and potato early dying. (Abstr.) Phytopathology 75:1279.

de Mendiburu, F. 2017. agricolae: Statistical Procedures for Agricultural Research. R package version 1.2-8. https://cran.r-project.org/web/packages/ agricolae/index.html

Dobinson, K. F. 1995. Genetic transformation of the vascular wilt fungus Verticillium dahliae. Can. J. Bot. 73:710-715.

Dung, J. K. S., Peever, T. L., and Johnson, D. A. 2013. Verticillium dahliae populations from mint and potato are genetically divergent with predominant haplotypes. Phytopathology 103:445-459. 
Gurung, S., Short, D. P. G., Atallah, Z. K., and Subbarao, K. V. 2014. Clonal expansion of Verticillium dahliae in lettuce. Phytopathology 104:641-649.

Hastie, A. C. 1964. The parasexual cycle in Verticillium albo-atrum. Genet. Res. 5:305-315.

Hastie, A. C. 1973. Hybridization of Verticillium albo-atrum and Verticillium dahliae. Trans. Br. Mycol. Soc. 60:511-523, IN9.

Inderbitzin, P., Davis, R. M., Bostock, R. M., and Subbarao, K. V. 2011. The Ascomycete Verticillium longisporum is a hybrid and a plant pathogen with an expanded host range. PLoS One 6:e18260.

Ishikawa, F. H., Souza, E. A., Shoji, J.-y., Connolly, L., Freitag, M., Read, N. D., and Roca, M. G. 2012. Heterokaryon incompatibility is suppressed following conidial anastomosis tube fusion in a fungal plant pathogen. PLoS One 7:e31175.

Jiménez-Díaz, R. M., Olivares-García, C., Landa, B. B., Jiménez-Gasco, M. M., and Navas-Cortés, J. A. 2011. Region-wide analysis of genetic diversity in Verticillium dahliae populations infecting olive in southern Spain and agricultural factors influencing the distribution and prevalence of vegetative compatibility groups and pathotypes. Phytopathology 101:304-315.

Jiménez-Gasco, M. M., Malcolm, G. M., Berbegal, M., Armengol, J., and Jiménez-Díaz, R. M. 2014. Complex molecular relationships between vegetative compatibility groups (VCGs) in Verticillium dahliae: VCGs do not always align with clonal lineages. Phytopathology 104:650-659.

Joaquim, T. R., and Rowe, R. C. 1990. Reassessment of vegetative compatibility relationships among strains of Verticillium dahliae using nitratenonutilizing mutants. Phytopathology 80:1160-1166.

Kabir, Z., Bhat, R. G., and Subbarao, K. V. 2004. Comparison of media for recovery of Verticillium dahliae from soil. Plant Dis. 88:49-55.

Katan, T. 2000. Vegetative compatibility in populations of Verticillium-An overview. Pages 69-86 in: Advances in Verticillium: Research and Disease Management. E. C. Tjamos, R. C. Rowe, J. B. Heale, and D. R. Fravel, eds. American Phytopathological Society Press, St. Paul, MN, U.S.A.

Klosterman, S. J., Atallah, Z. K., Vallad, G. E., and Subbarao, K. V. 2009. Diversity, pathogenicity and management of Verticillium species. Annu. Rev. Phytopathol. 47:39-62.

Klosterman, S. J., Subbarao, K. V., Kang, S., Veronese, P., and Gold, S. E. 2011. Comparative Genomics Yields Insights into Niche Adaptation of Plant Vascular Wilt Pathogens. PLoS Pathog 7:e1002137.

Korolev, N., Encarnación, P.-A., Bejarano-Alcázar, J., Rodríguez-Jurado, D., Katan, J., Katan, T., and Jiménez-Díaz, R. M. 2001. Comparative study of genetic diversity and pathogenicity among populations of Verticillium dahliae from cotton in Spain and Israel. Eur. J. Plant Pathol. 107:443-456.

Malcolm, G. M., Kuldau, G. A., Gugina, B. K., and Jiménez-Gasco, M. M. 2013. Hidden host plant associations of soilborne fungal pathogens: An ecological perspective. Phytopathology 103:538-544.

Matta, A., and Garibaldi, A. 1977. Control of Verticillium wilt of tomato by preinoculation with avirulent fungi. Neth. J. Plant Pathol.: 457-462.

Milgroom, M. G. 2015. Population Biology of Plant Pathogens: Genetics, Ecology, and Evolution. The American Phytopathological Society, St. Paul, MN, U.S.A.

Milgroom, M. G., Jiménez-Gasco, M.-M., Olivares-García, C., Drott, M. T., and Jiménez-Díaz, R. M. 2014. Recombination between clonal lineages of the asexual fungus Verticillium dahliae detected by genotyping by sequencing. PLoS One 9:e106740.

Powelson, M. L., and Rowe, R. C. 1993. Biology and management of early dying of potatoes. Annu. Rev. Phytopathol. 31:111-126.

Price, D., and Sackston, W. E. 1983. Cross protection against Verticillium in sunflower. (Abstr). Phytopathology 73:372.

Price, D., and Sackston, W. E. 1989. Cross protection among strains of Verticillium dahliae on sunflower. Pages 229-235 in: Vascular Wilt Disease of Plants. E. C. Tjamos and C. Beckman, eds. Springer, Berlin.

Qin, Q.-M., Vallad, G. E., and Subbarao, K. V. 2008. Characterization of Verticillium dahliae and $V$. tricorpus isolates from lettuce and artichoke. Plant Dis. 92:69-77.

Rafiei, V., Banihashemi, Z., Baustista-Jalon, L. S., Jiménez-Gasco, M. M., Turgeon, B. G., and Milgroom, M. G. 2018. Population genetics of Verticillium dahliae in Iran based on microsatellite and single nucleotide polymorphism. Phytopathology 108:780-788.

Robinson, N., Platt, H. W., and Lawrence, R. H. 2007. Interaction of various Verticillium species in combination with $V$. albo-atrum on Verticillium wilt disease development in potato. Am. J. Potato Res. 84: Article 133.

Shaner, G., and Finney, R. E. 1977. The effect of nitrogen fertilization on the expression of slow-mildewing resistance in Knox wheat. Phytopathology 67:1051-1056

Shittu, H. O., Castroverde, D. C. M., Nazar, R. N., and Robb, J. 2009. Plantendophyte interplay protects tomato against a virulent Verticillium. Planta 229:415-426.

Short, D. P. G., Gurung, S., Gladieux, P., Inderbitzin, P., Atallah, Z. K., Nigro, F., Li, G., Benlioglu, S., and Subbarao, K. V. 2015. Globally invading populations of the fungal plant pathogen Verticillium dahliae are dominated by multiple divergent lineages. Environ. Microbiol. 17: 2824-2840.

Short, D. P. G., Gurung, S., Hu, X., Inderbitzin, P., and Subbarao, K. V. 2014. Maintenance of Sex-Related Genes and the Co-occurrence of both mating types in Verticillium dahliae. PLoS One 9:e112145.

Strom, N. B., and Bushley, K. E. 2016. Two genomes are better than one: History, genetics, and biotechnological applications of fungal heterokaryons. Fungal Biol. Biotechnol. 3:4.

Tyvaert, L., França, S. C., Debode, J., and Höfte, M. 2014. The endophyte VerticilliumVt305 protects cauliflower against Verticillium wilt. J. Appl. Microbiol. 116:1563-1571.

Usami, T., Itoh, M., and Amemiya, Y. 2009. Asexual fungus Verticillium dahliae is potentially heterothallic. J. Gen. Plant Pathol. 75:422-427.

Wheeler, D. L., and Johnson, D. A. 2016. Verticillium dahliae infects, alters plant biomass, and produces inoculum on rotation crops. Phytopathology 106:602-613.

Wheeler, D. L., Dung, J. K. S., and Johnson, D. A. 2019. From pathogen to endophyte: An endophytic population of Veriticillium dahliae evolved from a sympatric pathogenic population. New Phytol. doi: 10.1111/nph.15567

Wilhelm, S. 1955. Longevity of Verticillium wilt fungus in the laboratory and field. Phytopathology 45:180-181. 